\title{
Extraction of Texture Features using GLCM and Shape Features using Connected Regions
}

\author{
Shijin Kumar P.S ${ }^{\# 1}$, Dharun V.S ${ }^{* 2}$ \\ "Research Scholar, Department of Electronics and Communication Engineering, \\ Noorul Islam University, Kumaracoil, Tamilnadu, India. \\ * Principal, Archana College of Engineering,Palamel, Nooranad, Alappuzha, Kerala, India. \\ 1shijinkumarps@yahoo.com \\ 2 dharunvs@yahoo.com
}

\begin{abstract}
Feature extraction is an important step in Computer Assisted Diagnosis of brain abnormalities using Magnetic Resonance Images (MRI).Feature Extraction is the process of reducing the size of image data by obtaining necessary information from the segmented image. The visual content of a segmented image can be captured using this process. From the extracted features it is possible to demarcate between normal and abnormal brain MRI. The reliability of the classification algorithm depends on segmentation method and extracted features. In this work texture features are extracted using Gray Level Co-occurrence Matrix (GLCM) and shape features are extracted using connected regions. Images with malignant tumor, benign tumor and normal brain have different features. This variation in feature values is useful in classification of MR images. The features thus obtained will be given to a classifier for training and testing.
\end{abstract}

Keywords - Brain tumor, Magnetic resonance imaging (MRI), Computer aided diagnosis (CAD), Feature Extraction, Gray Level Co-occurrence Matrix (GLCM), Connected Regions.

\section{INTRODUCTION}

Images obtained from an MRI Scanner are verified by a radiologist for the identification of abnormalities. Manual classification of MR images is a time consuming and challenging task [8]. Hence there is a necessity for a computer aided automatic tumor classification system. Major step involved in computer aided detection of brain abnormalities are pre-processing, segmentation, feature extraction and classification.After segmenting the tumor regions from the MRI, the features of the segmented regions are analysed. The properties which provide description about the whole image are called features [3]. Feature extraction process reduces the original MRI data set into a set of features. This feature set is also known a feature vector. These feature vectors are the basic inputs for any classification algorithm. Based on the severity of tumors, they are classified into Benign and Malignant [7]. Benign tumors are slow growing and less harmful tumors. Malignant tumors grow fast and affect surrounding tissues [7]. Particular features exhibited by these tumors are useful in the classification process.

Rajesh et al [9] used rough set theory for the extraction of features. The features thus extracted provided a classification efficiency of 90\%.Hiremath et al [13] introduced a feature extraction technique based on complementary wavelet transform. In this method features are extracted from 4 sub bands and the efficiency is more compared to features from a single band. Discrete Wavelet Transform (DWT) was used and the accuracy of classification is less compared to other methods. Huang et al [14] introduced a method for dimensionality reduction using sub band grouping and selection. Low classification accuracy and less efficiency are the demerits of this method.Ramteke et al [10] obtained statistical texture features from input dataset. The classification efficiency obtained was $80 \%$. Xuan et al [12] used texture symmetry and intensity based features are extracted from given MR images. The average accuracy was $96.02 \%$. Othman et al [11] used principal component analysis (PCA) for the extraction of features. The classification accuracy obtained was $96.33 \%$.

In this paper texture features are extracted using Gray Level Co-occurrence Matrix (GLCM) [2]and shape features are extracted using connected regions. The proposed methodology for feature extraction is described in section II. Results obtained by giving different input images are given is section III. Finally conclusion is providedin section IV.

\section{Methodology}

Brain MRI database is created using images collected from publicly available MRI images [17] and from various diagnosis centres in Kerala, India. These images are stored as gray scale images having size 256x256. Input MRI brain images are shown in Fig 1. 


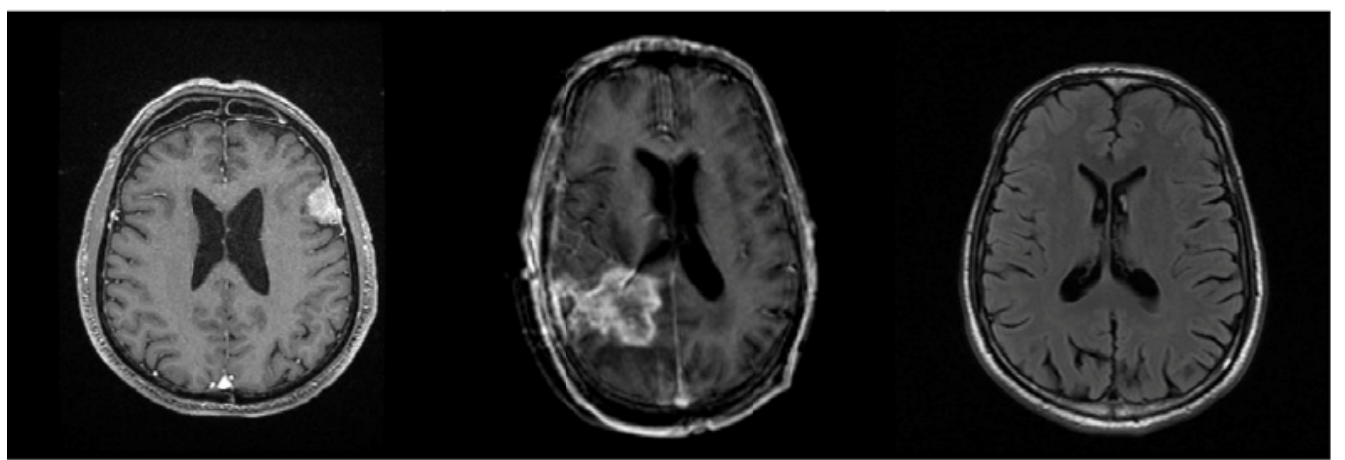

(a)

(b)

(c)

Fig.1. MRI ofhuman brain (a) with benign tumor, (b) with malignant tumor, (c) without tumor.

Initially the image is pre-processed to obtain noise free, enhanced and skull stripped brain MRI. Skull stripping is done using connected neighbours and morphological operation [15]. The removal of skull region helps to increase the efficiency of segmentation algorithm and can avoid misclassification.The skull stripped MR image thus obtained is segmented using a novel hybrid method based on the combination of K-means clustering algorithm and Texture Pattern Matrix [16]. Texture Pattern Matrix is a kernel based approach to consider the texture properties while performing segmentation. This method provides better accuracy and specificity compared to other methods.

\section{A. Extraction of Texture Features using GLCM}

Gray Level Co-occurrence Matrix (GLCM) is used to calculate the spacial dependence of gray levels in an image [2]. In GLCM the number of rows and columns are exactly equal to the number of gray levels in the image. Co-occurrence matrices are constructed in four spacial orientations $\left(0^{\circ}, 45^{\circ}, 90^{\circ}\right.$ and $\left.135^{\circ}\right)$. Another matrix is constructed as the average of preceding matrices. Let the Co-occurrence matrix be $P_{i, j}$ and the size of the matrix is $N \mathrm{x} N$. Each element $(i, j)$ represents the frequency by which pixel with gray level $i$ is spatially related to pixel with gray level $j$. Construction of GLCM from a gray scale image [5] is illustrated in Fig.2.

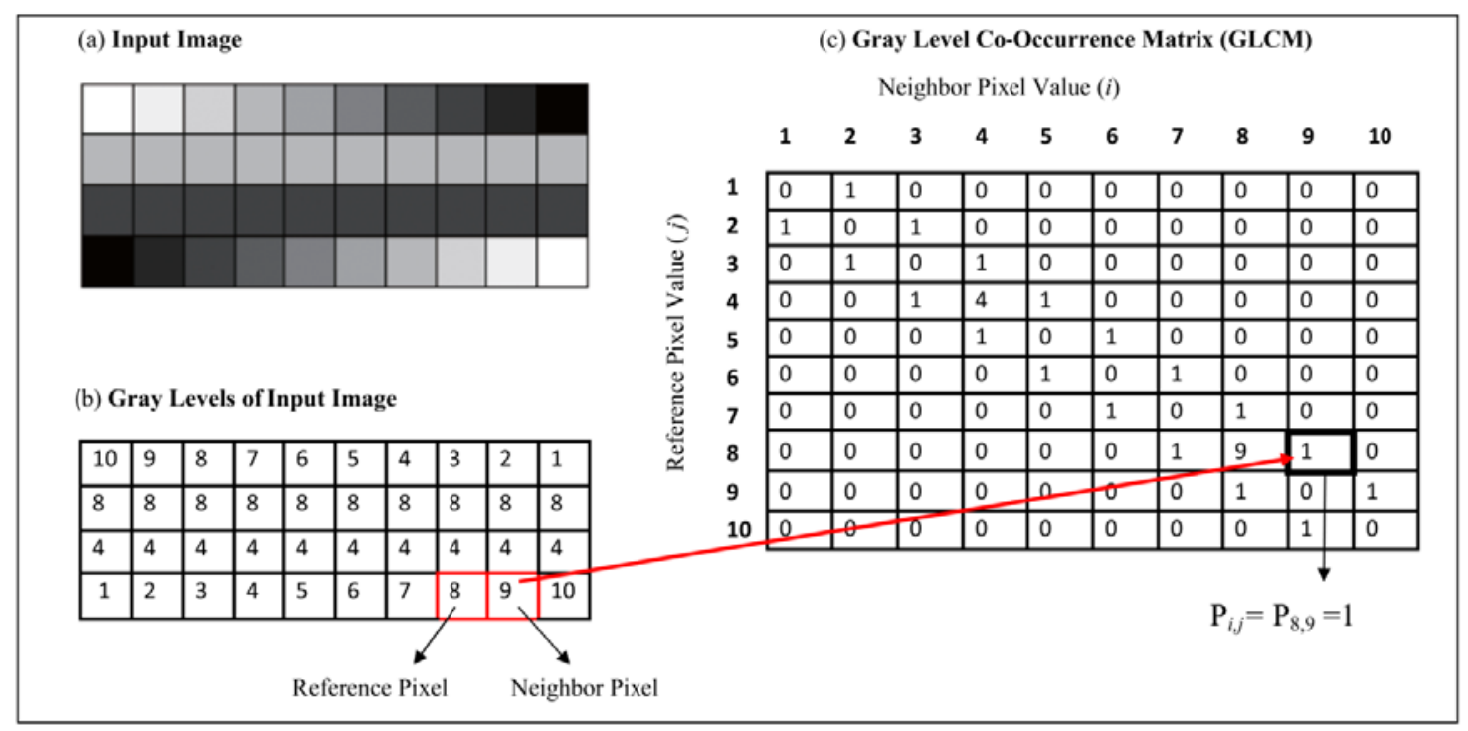

Fig.2. Construction of Gray Level Co-occurrence Matrix (GLCM) from an image.

The sample input image consists of 10 gray levels. GLCM represents the relation between reference pixel (i) and neighbour pixel $(j)$ in various orientation. Here the relation between pixels is calculated horizontally towards the right $\left(0^{\circ}\right)$. Initially, the value of each elements in GLCM $(i, j)$ is zero. The value of each element is updated as per the occurrence of pixels together.Texture features calculated using GLCM are Contrast, Correlation, Dissimilarity, Energy, Entropy, Homogeneity, Mean, Variance and Standard Deviation. The formulas for calculation of these features [6] are given in Table I. 
TABLE I. Formulas to calculate Texture Features from GLCM

\begin{tabular}{|c|c|c|}
\hline Sl.No & GLCM Feature & Formula \\
\hline 1. & Contrast & $\sum_{i, j=0}^{N-1} P_{i, j}(i-j)^{2}$ \\
\hline 2. & Correlation & $\sum_{i, j=0}^{N-1} P_{i, j}\left[\frac{\left(i-\mu_{i}\right)\left(j-\mu_{j}\right)}{\sqrt{\left(\sigma_{i}^{2}\right)\left(\sigma_{j}^{2}\right)}}\right]$ \\
\hline 3. & Dissimilarity & $\sum_{i, j=0}^{N-1} P_{i, j}|i-j|$ \\
\hline 4. & Energy & $\sum_{i, j=0}^{N-1} P_{i, j}^{2}$ \\
\hline 5. & Entropy & $\sum_{i, j=0}^{N-1} P_{i, j}\left(-\ln P_{i, j}\right)$ \\
\hline 6. & Homogeneity & $\sum_{i, j=0}^{N-1} \frac{P_{i, j}}{1+(i-j)^{2}}$ \\
\hline 7. & Mean & $\mu_{i}=\sum_{i, j=0}^{N-1} i\left(P_{i, j}\right), \quad \mu_{j}=\sum_{i, j=0}^{N-1} j\left(P_{i, j}\right)$ \\
\hline 8. & Variance & $\sigma_{i}^{2}=\sum_{i, j=0}^{N-1} P_{i, j}\left(i-\mu_{i}\right)^{2}, \sigma_{j}^{2}=\sum_{i, j=0}^{N-1} P_{i, j}\left(j-\mu_{j}\right)^{2}$ \\
\hline 9. & Standard Deviation & $\sigma_{i}=\sqrt{\sigma_{i}^{2}}, \quad \sigma_{j}=\sqrt{\sigma_{j}^{2}}$ \\
\hline
\end{tabular}

B. Extraction of Shape Features using Connected Regions

Shape is a binary representation of extend of the object. Shape features refer to the geometric properties of an object and the external boundary is used to calculate these features. Area, Perimeter and Circularity are the major shape features we calculate in our method. Extracted shape features can provide powerful information for image classification. The shape features varies for different tumor types. Shape features are calculated using connected regions in the image. Boundary pixels are used to calculate the perimeter and area. Circularity is calculated using area and perimeter. The formulas used to calculate shape features are given in Table II.

$E_{d}(i, j)$ is the boundary pixels of the region.

$\mathrm{b}(i, j)= \begin{cases}1, & f(i, j) \geq T_{h} \\ 0, & f(i, j)<T_{h}\end{cases}$

$$
\mathrm{E}_{\mathrm{d}}(\mathrm{i}, \mathrm{j})= \begin{cases}1, & \text { if Connectivity of } b(i, j)==2 \\ 0, & \text { else }\end{cases}
$$

$T_{h}=\frac{1}{2}[\max f(i, j)-\min f(i, j)]$ 
TABLE II. Formulas to calculate Shape Features using Connected Regions

\begin{tabular}{|l|c|c|}
\hline Sl.No & Shape Feature & Formula \\
\hline 1. & Perimeter (P) & $\sum_{i, j=0}^{M, N} E_{d}(i, j)$ \\
\hline 2. & Area (A) & $\sum^{M, N} b(i, j)$ \\
\hline 3. & Circularity (C) & $\frac{4 \pi A}{P^{2}}$ \\
\hline
\end{tabular}

\section{III.RESULTS AND DISCUSSION}

In this paper the methods used for feature extraction are Grey Level Co-occurrence Matrix (GLCM) and Connected Regions. During the experiment 3 images are taken from the MR image dataset. First one is an MRI with benigntumor (grade I and grade II), second one is an MRI with malignant tumor (grade III and grade IV) and the third one is a normal MRI (without tumor). Each image is segmented and the Grey Level Co-occurrence Matrix (GLCM) is created. From the GLCM, texture features are calculatedand connected regions are used to extract shape features. The feature values obtained from each images are shown in Table III. Malignant and benign MRI produces finite values for circularity, area and perimeter. Since there is no tumor present in normal brain MRI, the values for circularity, area and perimeter are 0. The features are calculated accurately using GLCM.

TABLE III. TEXTURE FEATURES AND SHAPE FEATURES EXTRACTED USING GLCM AND CONNECTED REGIONS

\begin{tabular}{|l|l|l|l|}
\hline Features & Malignant Tumor & Benign Tumor & Normal Brain \\
\hline Contrast & 61.7289 & 112.1709 & 15.9206 \\
\hline Correlation & 0.8643 & 0.9297 & 0.6488 \\
\hline Dissimilarity & 0.5156 & 0.7566 & 0.0797 \\
\hline Energy & 0.9789 & 0.9547 & 0.9987 \\
\hline Entropy & 0.1250 & 0.2490 & 0.0069 \\
\hline Homogeneity & 0.9900 & 0.9796 & 0.9994 \\
\hline GLCM mean & 2.4098 & 5.1603 & 1.1015 \\
\hline Variance & 227.4835 & 797.8255 & 22.6651 \\
\hline Standard Deviation & 15.0826 & 28.2458 & 4.7608 \\
\hline Area & 136.5000 & 492 & 0 \\
\hline Perimeter & 132 & 488 & 0 \\
\hline Circularity & 0.0984 & 0.0260 & 0 \\
\hline
\end{tabular}

IV.CONCLUSION

We have used nearly 300 brain MR images for our work. The proposed feature extraction method is faster and less complex compared to existing methods. The accuracy is high and the obtained features are authentic. Texture features are extracted using GLCM and shape features are extracted using connected regions. The shape features are extracted directly from the segmented image. It is the simplest and less complex method for the calculation of texture and shape features.These features can be used for training the classification algorithm.Different set of features are obtained for malignant, benign and normal MRI. The accuracy of classification can be increased by using larger dataset. Our future goal is to develop a classification algorithm based on artificial neural network and to categorize input brain MR images. 


\section{REFERENCES}

[1] Ruan, S., Jaggi, C., Xue, J., Fadili, J. \&Bloyet, D. Brain Tissue Classification of Magnetic Resonance Images Using Partial Volume Modeling. IEEE Transactions onMedical Imaging, Vol.19, No.12, pp.1179-1187, 2000.

[2] V. Vijayakumar, V. Neelanarayanan, A. Veeramuthu, S. Meenakshi, V. PriyaDarsini, Big Data, Cloud and Computing ChallengesBrain Image Classification Using Learning Machine Approach and Brain Structure Analysis, Procedia Computer Science, Volume 50, 2015, Pages 388-394, ISSN 1877-0509,

[3] Robert. M. Haralick, K. Shanmugam and I. Dinstein, "Textural Features for Image Classification," in IEEE Transactions on Systems, Man, and Cybernetics, vol. SMC-3, no. 6, pp. 610-621, Nov. 1973.

[4] Shijin, K.P.S.; Edwin, D.D., "Simulated attack based feature region selection for efficient digital image watermarking," in Computing, Electronics and Electrical Technologies (ICCEET), 2012 International Conference on , vol., no., pp.1128-1132, 21-22

[5] Mattonen, S., Huang, K., Ward, A., Senan S, Palma, D, "New techniques for assessing response after hypofractionated radiotherapy for lung cancer" in Journal of Thoracic Disease, Vol.6, No.4, 2014.

[6] Robert M. Haralick, "Statistical and structural approaches to texture," Proc. IEEE, vol. 67, no. 5, pp. 786-804, 1979.

[7] Shijin Kumar P S and Dharun V S, A Study of MRI Segmentation Methods in Automatic Brain Tumor Detection. International Journal of Engineering and Technology. 2016 April-May; 8 (02):609-614.

[8] Kauset.al., “ Automated segmentation of MR imagesof brain tumors”, Journal of radiology, vol.218, no.2,pp: 586-591, 2001.

[9] T. Rajesh, R. Suja Mani Malar," Rough Set Theory and Feed Forward Neural Network Based Brain Tumor Detection in Magnetic Resonance Images",IEEE International Conference on Advanced Nanomaterials\& Emerging Engineering Technologies, 2013.

[10] R. J. Ramteke1, KhachaneMonali Y., " Automatic Medical Image Classification and Abnormality Detection Using K-Nearest Neighbour" , International Journal of Advanced Computer Research,Volume-2 Number-4 Issue-6 December-2012.

[11] MohdFauzi Othman, MohdAriffanan, MohdBasri, "Probabilistic Neural Network for Brain Tumor Classification" ,IEEE International Conference on Intelligent Systems, Modelling and Simulation,2011.

[12] Xiao Xuan, Qingmin Liao, Statistical Structure Analysis in MRI Brain Tumor Segmentation" ,IEEE International Conference on Image and Graphics, 2007.

[13] Hiremath P, Shivashankar S. Wavelet basedfeatures for texture classification. Graphics,Vision and Image Processing Journal;6:558.2006.

[14] Huang K, Aviyente S. Wavelet featureselection for image classification. IEEE Transaction on Image Processing; 17:1709-20, 2008.

[15] Shijin Kumar P S and Dharun V S, An efficient skull stripping algorithm using connected regions and morphological operation. ARPN Journal of Engineering and Applied Sciences. 2016 April; 11 (07):4305-4309.

[16] Shijin Kumar P S and Dharun V S, Hybrid Brain MRI SegmentationAlgorithm Based on K-means Clustering and Texture Pattern Matrix. International Journal of Applied Engineering Research. Volume 11,No. 6 (2016)Pages: 4343-4348.

[17] Brain MRI database available on: https://radiopaedia.org/encyclopaedia/cases/central-nervous-system.

\section{AUTHOR PROFILE}

Shijin Kumar P.S. received the B.Tech. degree in Electronics and Communication Engineering from University of Kerala, India, in 2006, the M.E. degree in Communication Systems from Anna University, Tirunelveli, Tamil Nadu, India, in 2009. He is currently working toward the Ph.D. degree in Electronics and Communication Engineering at Noorul Islam University, Tamil Nadu, India. His current research interest includes image processing focusing on medical images, automatic segmentation of brain tumors in MR Images, Image Classification etc. He is currently an Assistant Professor in the department of Electronics and Communication Engineering at PRS College of Engineering and Technology, Paliyode, Trivandrum, Kerala, India. He was an Assistant Professor in the department of Electronics and Communication Engineering at Marthandam College of Engineering and Technology, Kuttakuzhi, Kanyakumari district, Tamil Nadu, India.

Dharun V.S. received the B.E. degree in Electrical and Electronics Engineering from Bharathiar University, Tamil Nadu, India, in 2002, the M.E. degree in Applied Electronics from Anna University, Chennai, Tamil Nadu, India, in 2004, the Ph.D. degree in Applied Electronics/ Computer Science and Engineering from ManonmaniamSundaranar University, Tamil Nadu, India, in 2013. His current research interest includes speech processing, image processing focusing on medical images, automatic segmentation of brain tumors in MR Images, image classification etc. He is currently the Principal ofArchana College of Engineering,Palamel, Nooranad, Alappuzha, Kerala, India. He was the Head and Professor in the department of Electronics and Communication Engineering at MET'S School of Engineering, Mala, Thrissur, Kerala, India. He was an Associate Professor and Head in the department of Biomedical Engineering at Noorul Islam University, Tamil Nadu, India. 